\title{
Prospective Registry Study of Primary Dyslipidemia (PROLIPID): Rationale and Study Design
}

\author{
Hayato Tada ${ }^{1}$, Tomoyuki Kurashina ${ }^{2}$, Masatsune Ogura ${ }^{3}$, Misa Takegami ${ }^{4}$, Yoshihiro Miyamoto ${ }^{5}$, \\ Hidenori Arai ${ }^{6}$, Mariko Harada-Shiba ${ }^{7}$, Shun Ishibashi ${ }^{2},{ }^{+}$Committee on Primary Dyslipidemia under the \\ Research Program on Rare and Intractable Disease of the Ministry of Health, Labour and Welfare of Japan
}

\footnotetext{
${ }^{1}$ Department of Cardiovascular Medicine, Kanazawa University Graduate School of Medical Sciences, Kanazawa, Japan

${ }^{2}$ Division of Endocrinology and Metabolism, Department of Internal Medicine, School of Medicine, Jichi Medical University, Tochigi, Japan

${ }^{3}$ Department of General Medical Science, Chiba University Graduate School of Medicine, Chiba, Japan

${ }^{4}$ Department of Preventive Medicine and Epidemiology, National Cerebral and Cardiovascular Center Research Institute, Osaka, Japan ${ }^{5}$ Department of Preventive Cardiology, National Cerebral and Cardiovascular Center, Osaka, Japan

${ }^{6}$ National Center for Geriatrics and Gerontology, Aichi, Japan

${ }^{7}$ Department of Molecular Pathogenesis, National Cerebral and Cardiovascular Center Research Institute, Osaka, Japan
}

${ }^{\dagger}$ The Committee on Primary Dyslipidemia under the Research Program on Rare and Intractable Disease of the Ministry of Health, Labour and Welfare of Japan: Mariko Harada-Shiba (Department of Molecular Pathogenesis, National Cerebral and Cardiovascular Center Research Institute, Osaka, Japan), Shun Ishibashi (Division of Endocrinology and Metabolism, Department of Internal Medicine, School of Medicine, Jichi Medical University, Tochigi, Japan), Shinji Yokoyama (Institute for Biological Functions, Chubu University, Aichi, Japan), Hitoshi Shimano (Department of Internal Medicine (Endocrinology and Metabolism, Ibaraki, Japan) Faculty of Medicine University of Tsukuba, Tsukuba, Japan), Koutaro Yokote (Department of Endocrinology, Hematology and Gerontology, Chiba University Graduate School of Medicine, Chiba, Japan), Hideaki Bujo (Department of Clinical-Laboratory and Experimental-Research Medicine, Toho University Sakura Medical Center, Chiba, Japan), Shizuya Yamashita (Department of Cardiology, Rinku General Medical Center, Osaka, Japan), Kazuhisa Tsukamoto (Department of Internal Medicine, Teikyo University, Tokyo, Japan), Toshio Hayashi (School of Health Sciences, Nagoya University Graduate School of Medicine, Nagoya, Japan), Katsunori Ikewaki (Division of Neurology, Anti-Aging, and Vascular Medicine, Department of Internal Medicine, National Defense Medical College, Saitama, Japan), Takanari Gotoda (Department of Metabolic Biochemistry, Faculty of Medicine, Kyorin University, Tokyo, Japan), Kazushige Dobashi (Department of Pediatrics, School of Medicine, University of Yamanashi, Yamanashi, Japan), Yoshihiro Miyamoto (Open Innovation Center, National Cerebral and Cardiovascular Center, Osaka, Japan), Yoshitaka Iwanaga, (Center for Cerebral and Cardiovascular Disease Information, National Cerebral and Cardiovascular Center, Osaka, Japan), Misa Takegami (Department of Preventive Medicine and Epidemiology, National Cerebral and Cardiovascular Center, Osaka, Japan), Yoshiki Sekijima (Department of Medicine (Neurology \& Rheumatology), Shinshu University School of Medicine, Matsumoto, Japan), Yasushi Ishigaki (Division of Diabetes, Metabolism and Endocrinology, Department of Internal Medicine, Iwate Medical University, Iwate, Japan), Hiroaki Okazaki (Department of Diabetes and Metabolic Diseases, Graduate School of Medicine, The University of Tokyo, Tokyo, Japan), Atsushi Nohara (Ishikawa Prefectural Central Hospital, Kanazawa, Japan), Shingo Koyama (Division of Neurology and Clinical Neuroscience, Department of Internal Medicine III, Yamagata University Faculty of Medicine, Yamagata, Japan), Kyoko Inagaki (Division of Diabetes, Endocrinology, and Metabolism, Department of Medicine, Nippon Medical School, Tokyo, Japan), Koh Ono (Department of Cardiovascular Medicine, Kyoto University Graduate School of Medicine, Kyoto, Japan), Masahiro Koseki (Division of Cardiovascular Medicine, Department of Medicine, Osaka University Graduate School of Medicine, Osaka, Japan), Hiroyuki Daida (Faculty of Health Science, Juntendo University , Juntendo University Graduate School of Medicine, Tokyo, Japan), Manabu Takahashi (Division of Endocrinology and Metabolism, Department of Internal Medicine, Jichi Medical University, Tochigi, Japan), Kimitoshi Nakamura (Department of Pediatrics, Kumamoto University Graduate School of Medical Sciences, Kumamoto, Japan), Takashi Miida (Department of Clinical Laboratory Medicine, Juntendo University Graduate School of Medicine, Tokyo, Japan), Masa-aki Kawashiri (Department of Cardiovascular Medicine, Graduate School of Medical Sciences, Kanazawa University, Kanazawa, Japan), Tetsuo Minamino (Department of Cardiorenal and Cerebrovascular Medicine, Faculty of Medicine, Kagawa University, Kagawa, Japan), Sachiko Okazaki (Division for Health Service Promotion, The University of Tokyo, Tokyo, Japan), Hayato Tada (Department of Cardiovascular Medicine, Kanazawa University Graduate School of Medical Sciences, Kanazawa, Japan), Jun Wada (Department of Nephrology, Rheumatology, Endocrinology and Metabolism, Okayama University Graduate School of Medicine, Dentistry and Pharmaceutical Sciences, Okayama, Japan), Masatsune Ogura (Department of Metabolism and Endocrinology, Eastern Chiba Medical Center, Togane, Japan), Hiroshi Yoshida (Department of Laboratory Medicine, The Jikei University Kashiwa Hospital, Chiba, Japan), Yu Kataoka (Department of Cardiovascular Medicine National Cerebral and Cardiovascular Center, Osaka, Japan), Hirotoshi Ohmura (Department of Cardiovascular Medicine, School of Medicine, Juntendo University, Tokyo, Japan), Mika Hori (Department of Endocrinology, Research Institute of Environmental 
Medicine, Nagoya University, Nagoya, Japan), Kota Matsuki (Department of Endocrinology and Metabolism, Hirosaki University Graduate School of Medicine, Hirosaki, Japan), Masashi Yamamoto (Department of Endocrinology, Hematology and Gerontology, Chiba University Graduate School of Medicine, Chiba, Japan), Yasuo Takeuchi (Division of Nephrology, Kitasato University School of Medicine, Kanagawa, Japan), Atsuko Nakatsuka (Department of Nephrology, Rheumatology, Endocrinology and Metabolism, Okayama University Graduate School of Medicine, Dentistry and Pharmaceutical Sciences, Okayama, Japan), Daisaku Masuda (Department of Cardiology, Health Care Center, Rinku Innovation Center for Wellness Care and Activities (RICWA), Rinku General Medical Center, Osaka, Japan), Satoshi Hirayama (Department of Clinical Laboratory Medicine, Juntendo University Graduate School of Medicine, Tokyo, Japan), Masayuki Kuroda (Center for Advanced Medicine, Chiba University Hospital, Chiba University, Chiba, Japan), Takashi Yamaguchi (Center of Diabetes, Endocrinology and Metabolism, Toho University Sakura Medical Center, Chiba, Japan), Takeyoshi Murano (Clinical Laboratory Program, Faculty of Science, Toho University, Chiba, Japan), Hirofumi Okada (Department of Cardiovascular Medicine, Kanazawa University Graduate School of Medical Sciences, Kanazawa, Japan), Manabu Minami (Department of Data Science, National Cerebral and Cardiovascular Center, Osaka, Japan), Hisashi Makino (Division of Atherosclerosis and Diabetes, National Cerebral and Cardiovascular Center, Osaka, Japan), Masaki Matsubara (Division of Atherosclerosis and Diabetes, National Cerebral and Cardiovascular Center, Osaka, Japan), Sayaka Funabashi (Department of Cardiovascular Medicine, Kyorin University Hospital, Tokyo, Japan).

Introduction: Primary dyslipidemias are inherited disorders in plasma lipoprotein metabolism that lead to serious cardiovascular and other complications. The Japanese Ministry of Health, Labor and Welfare (MHLW) covers medical expenses, under the Research Program on Rare and Intractable Diseases, for homozygous familial hypercholesterolemia $(\mathrm{FH})$, familial chylomicronemia, sitosterolemia, cerebrotendinous xanthomatosis, lecithin:cholesterol acyltransferase deficiency, Tangier disease, and abetalipoproteinemia. Apolipoprotein A1 deficiency, heterozygous FH, and type III hyperlipoproteinemia are covered by the MHLW Pediatric Chronic Disease Program. Heterozygous FH and type III hyperlipoproteinemia are also important for their relatively common prevalence and, accordingly, high impact on Japanese public health by significant contribution to the overall prevalence of cardiovascular diseases. Therefore, a systemic survey of these diseases is mandatory to estimate their actual situation, such as prevalence, clinical manifestations, and prognoses among the Japanese population. The impact of these rare and intractable diseases on cardiovascular and other complications will likely be higher among Japanese people than other ethnicities because the general Japanese population has many cardioprotective aspects. The current study intends to conduct a multicenter registry of these diseases to assess their demographics and clinical features comprehensively.

Methods and Analysis: The Prospective Registry Study of Primary Dyslipidemia is a registry-based prospective, observational, multicenter cohort study in Japan, enrolling patients who fulfill the Japanese clinical criteria of the primary dyslipidemias listed above, from 26 participating institutes from August 2015 to March 2023. A total of 1,000 patients will be enrolled in the study and followed for 10 years. Clinical parameters are collected, including physical and laboratory findings, genetic analysis, drugs, lifestyle management, and clinical events, especially cardiovascular events. The primary endpoint of this study is the new onset of cardiovascular disease and acute pancreatitis, and the secondary endpoint is death from any causes.

Ethics and Dissemination: This study complies with the Declaration of Helsinki, the Ethical Guidelines for Medical and Health Research Involving Human Subjects, and all other applicable laws and guidelines in Japan. The institutional review boards have approved this study protocol at all participating institutes. The final results are to be published at appropriate international conferences and in peer-reviewed journals.

Trial registration: This study was registered at the University Hospital Medical Information Network (UMIN) Clinical Trials Registry (UMIN ID: UMIN000042782).

Key words: Intractable disease, Dyslipidemia, Genetics, Registry

Address for correspondence: Mariko Harada-Shiba, Department of Molecular Pathogenesis, National Cerebral and Cardiovascular Center Research Institute, 6-1 Kishibe-shinmachi, Suita, 564-8565, Japan. E-mail: mshiba@ncvc.go.jp

Received: August 17, $2021 \quad$ Accepted for publication: September 26, 2021

Copyright@2021 Japan Atherosclerosis Society

This article is distributed under the terms of the latest version of CC BY-NC-SA defined by the Creative Commons Attribution License.

\section{Advance Publication Journal of Atherosclerosis and Thrombosis}




\section{Introduction}

Molecular and genetic bases have been clarified for many primary dyslipidemias and monogenic inherited disorders of lipid and lipoprotein metabolism during the past decades. There have also been extensive efforts to improve their morbidity and mortality, including attempted gene therapies. However, many of them remain intractable, leaving patients to suffer from poor prognoses by their complications. Under these circumstances, the Japanese Ministry of Health, Labor and Welfare (MHLW) specified seven primary dyslipidemias were "rare and intractable diseases" to cover the cost of their medical care and alleviate patients' economic burden, including homozygous familial hypercholesterolemia (HoFH), sitosterolemia, cerebrotendinous xanthomatosis (CTX), lecithin cholesterol acyltransferase (LCAT) deficiency, Tangier disease, primary chylomicronemia, and abetalipoproteinemia. In addition, APOA1 deficiency, heterozygous $\mathrm{FH}$ $(\mathrm{HeFH})$, and type III hyperlipoproteinemia are covered under the MHLW program for Pediatric Chronic Diseases. Heterozygous FH and type III hyperlipoproteinemia are also important because of their relatively high prevalence among the general population and their substantial impact on public health in Japan due to their risk for cardiovascular complications. Both of the disorders are treatable to reduce cardiovascular risk and therefore should be paid more attention to from the public health viewpoint.

Since saving patients with rare and intractable diseases by clarifying the disease causes will surely lead to saving the general dyslipidemias caused by common genetic variations, it is important to investigate the real situation of these diseases across general populations. Some cross-sectional studies estimated the prevalence, clinical manifestations, and complications in Japanese patients with FH or CTX ${ }^{1,2)}$. However, these studies were underpowered because of their relatively small sample size, and cross-sectional nature of study design limits the power of assessments for their clinical events. In that sense, few longitudinal cohort studies have been performed in a large scale to find the current status, clinical features, and prognosis of the primary dyslipidemias in particular. A Prospective Registry Study of Primary Dyslipidemia (PROLIPID) was launched in 2015 to circumvent these issues, aiming to recruit at least 1,000 patients with the primary dyslipidemias specified above.

Familial hypercholesterolemia $(\mathrm{FH})$ is an autosomal dominant inherited disorder caused by mutations in the genes related to the LDL receptor pathway to clear plasma LDL. The prevalence of heterozygous $\mathrm{FH}(\mathrm{HeFH})$ has been estimated as 1 in 300 among general populations worldwide ${ }^{3,4)}$. HyperLDL-cholesterolemia, systemic xanthomas characterize heFH, and premature coronary artery disease, accounting for one of the major causes of premature $\mathrm{CVD}^{5)}$. HyperLDLemia in $\mathrm{HeFH}$ is mostly treatable by lifestyle management and/or drug therapy to reduce the CVD risk to a similar level to the general population if initiated early ${ }^{6,7)}$. The other common primary dyslipidemia is type III hyperlipoproteinemia. This disease is mainly caused by E2/E2 genotype in apolipoprotein $\mathrm{E}(A P O E)$ as 1 for 1,600 in the general Japanese population under certain nutritional conditions $^{8}$. It is known to be associated with elevated CVD risk mainly due to the elevation in remnant lipoprotein cholesterol ${ }^{9)}$. The risk of this type of dyslipidemia is also manageable by lifestyle control and drug regimens.

Pathogenic mutations in two alleles cause homozygous $\mathrm{FH}(\mathrm{HoFH})$, either in $\mathrm{LDL}$ receptor $(L D L R)$, apolipoprotein $\mathrm{B}(A P O B)$, proprotein convertase subtilisin/kexin type 9 (PCSK9), or LDLR adaptor protein 1 (LDLRAP1) or by the mutations combined in those genes and exhibits much severer clinical manifestation than $\mathrm{HeFH}$. The prevalence is estimated as 1 in $300,000^{10,11)}$. Timely diagnosis and commencement of LDL-lowering therapies would significantly reduce cardiovascular complication risk in FH patients ${ }^{12,13)}$. Similar to $\mathrm{HoFH}$, sitosterolemia is caused by double pathogenic mutations in ATPbinding cassette sub-family $\mathrm{G}$ member 5 (ABCG5) or ATP-binding cassette sub-family $\mathrm{G}$ member 8 $(A B C G 8)$ to exhibit systemic xanthomas and premature cardiovascular disease (CVD) associated with elevated LDL cholesterol and sitosterol ${ }^{14,15)}$. Double pathogenic mutations cause CTX in CYP27A to show very similar clinical manifestations that can be difficult to differentiate from $\mathrm{HoFH}$ and sitosterolemia. CTX is characterized by progressive cerebellar ataxia after puberty along with juvenile cataracts, chronic diarrhea of juvenile or infantileonset, childhood neurological deficit, and tendinous or tuberous xanthomas ${ }^{16)}$. In contrast, abetalipoproteinemia is characterized by extremely low LDL cholesterol and triglycerides, caused by double deleterious mutations in microsomal triglyceride transfer protein. Patients with this disorder typically suffer from complications such as spinocerebellar degeneration and pigmentary degeneration of the retina due to fat-soluble vitamin deficiencies ${ }^{17)}$.

Pathogenic mutations cause familial chylomicronemia in lipoprotein lipase $(L P L)$, or its regulators, apolipoprotein CII (APOC2), apolipoprotein A5 
(APOA5), glycosylphosphatidylinositol-anchored high-density lipoprotein binding protein 1 (GPIHBP1), and lipase maturation factor 1 (LMF1). The patients have severe hypertriglyceridemia and fasting chylomicronemia, and this phenotype predisposes the affected individuals to acute pancreatitis ${ }^{18)}$.

The other group of intractable dyslipidemia is a disorder of HDL metabolism; Tangier disease, lecithin cholesterol acyltransferase (LCAT) deficiency, and apolipoprotein A1 (APOA1) deficiency. Tangier disease is caused by double pathogenic mutations in ATP-binding cassette transporter A1 $(A B C A 1)$ and appears with an extremely low level of plasma HDL cholesterol. Patients with this disease develop massive cholesterol ester depositions in various organs, including tonsils, liver, and spleen. In addition, these patients often develop premature atherosclerosis caused by fatty deposits in the arteries ${ }^{19)}$. LCAT deficiency is caused by double pathogenic mutations in the LCAT gene and appears in two different forms. Familial LCAT deficiency is caused by complete loss of the LCAT activity, while in fish-eye disease, LCAT activity is partially deficient. LCAT is the enzyme that transfers acyl chain from the 2-position of lecithin to cholesterol on HDL to generate cholesterol acylester and leads HDL to "maturation." Clinical manifestations of this disease include diffuse corneal opacity, "target cell" hemolytic anemia, renal failure, atherosclerosis, hepatosplenomegaly, and enlarged lymph nodes, due to accumulated unesterified cholesterol $^{20)}$. APOA1 deficiency also exhibits extremely low levels of HDL cholesterol, caused by double pathogenic mutations in APOA1 gene. The manifestations of this disease include xanthomas, corneal opacity, and CVD ${ }^{21,22)}$.

Due to their rarity, the accumulation of the data of these intractable diseases is not adequate for their natural history, current clinical situations, and factors associated with prognosis. However, several large scale registry studies have clarified some useful clinical information and genetic backgrounds ${ }^{23-29)}$. Therefore, it is essential to understand the unique profiles of these diseases in Japan for frequency, phenotype, and genotype and their clinical prognosis, hopefully, based on the registration of all the patients, to help useful feedback to the current guidelines for their diagnosis and management. In addition, it is also important to investigate the real fact in Japan on heterozygous $\mathrm{FH}$ and type III hyperlipoproteinemia from the viewpoint of public health for their phenotype and genotype and clinical prognosis, in order to shape up the current guidelines and criteria to evaluate the socioeconomic value of treatment of these disorders.

Under these conditions, a nationwide, multicenter registry was organized by the Committee on Primary Dyslipidemia under the Research Program on Rare and Intractable Disease of the Ministry of Health, Labor and Welfare of Japan to comprehensively assess their phenotypes and genotypes. This should lead to a better understanding of these genetic disorders, not only for Japanese patients but also for patients elsewhere in the world.

\section{Methods}

\section{Study Design}

This nationwide, registry-based prospective, observational, multicenter cohort study starts from August 2015 to March 2023. Enrolled patients have primary dyslipidemias, including $\mathrm{FH}$ (homozygous or heterozygous), type III hyperlipoproteinemia, familial chylomicronemia, sitosterolemia, CTX, LCAT deficiency, Tangier disease, APOA1 deficiency, and abetalipoproteinemia who fulfill the clinical criteria of each disorder ${ }^{9-11,15-21)}$ from 26 participating institutes across Japan (Fig. 1). The patients are to be followed for 10 years. A total of 1,000 patients will be enrolled. This is based on the numbers of registered patients of intractable diseases in 2019 open to the public by MHLW of Japan ${ }^{30)}$, and those with other diseases who can register in this study. Fig. 1 shows the participating institutes. Participating institutes where the members and supporters of the Committee on Primary Dyslipidemia under the Research Program on Rare and Intractable Disease of the MHLW of Japan are working have been selected. Table 1 shows a schedule of the assessments and evaluations of this study. The primary outcome is the new onset of CVD events or acute pancreatitis. The secondary outcome is all causes of death.

The protocol of this study (version 9.0, dated 7 Feb 2020) has been approved by the Institutional Review Board at National Cerebral and Cardiovascular Center and all institutes participating in this study. In addition, this study complies with the Declaration of Helsinki, the Ethical Guidelines for Medical and Health Research Involving Human Subjects, and all other applicable laws and guidelines in Japan. It was registered at the University Hospital Medical Information Network (UMIN) Clinical Trials Registry (UMIN000042782).

\section{Study Participants}

Patients clinically diagnosed with each intractable disease are recruited from August 2015 to March 2023 and followed up over 10 years. The participants fulfilling both inclusion criteria participate in this study (Table 2). The participants with any of the

\section{Advance Publication Journal of Atherosclerosis and Thrombosis}




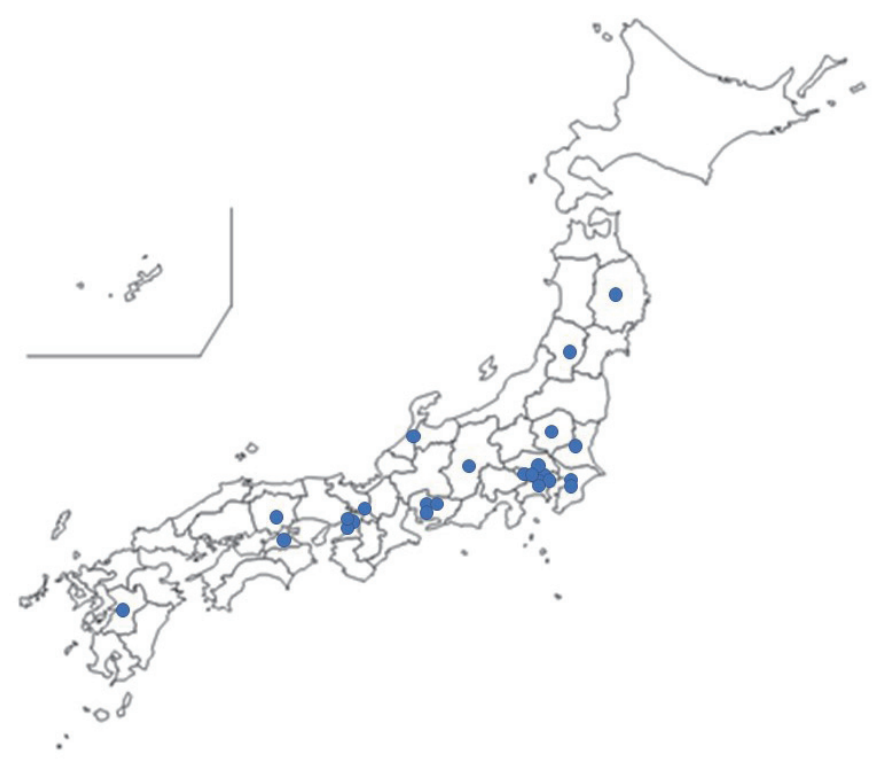

Fig. 1. Participating study centers, including 26 institutes across Japan

Blue circles indicate the institutes participating in this study.

Table 1. Assessments of this study

\begin{tabular}{|c|c|c|c|c|c|c|}
\hline Units & Baseline & $1 \mathrm{yr}$ & $2-4 \mathrm{yr}$ & $5 \mathrm{yr}$ & $6-9 \mathrm{yr}$ & $10 \mathrm{yr}$ \\
\hline Entry & $\mathrm{X}$ & & & & & \\
\hline Demographics & $\mathrm{X}$ & & & & & \\
\hline Untreated lipids & $\mathrm{X}$ & & & & & \\
\hline Blood tests & $\mathrm{X}$ & & & & & \\
\hline Physical and imaging & $\mathrm{X}$ & & & & & \\
\hline Family history & $\mathrm{X}$ & & & & & \\
\hline Medical history & $\mathrm{X}$ & & & & & \\
\hline Medicine & $\mathrm{X}$ & & & $\mathrm{X}$ & & $\mathrm{X}$ \\
\hline Lifestyle habits & $\mathrm{X}$ & & & & & \\
\hline DNA & $\mathrm{X}$ & & & & & \\
\hline Events (CVD) & & $\mathrm{X}$ & $\mathrm{X}$ & $\mathrm{X}$ & $\mathrm{X}$ & $\mathrm{X}$ \\
\hline Events (Stroke) & & $\mathrm{X}$ & $\mathrm{X}$ & $\mathrm{X}$ & $\mathrm{X}$ & $\mathrm{X}$ \\
\hline Events (valvular and aortic disease) & & $\mathrm{X}$ & $\mathrm{X}$ & $\mathrm{X}$ & $\mathrm{X}$ & $\mathrm{X}$ \\
\hline Events (acute pancreatitis) & & $\mathrm{X}$ & $\mathrm{X}$ & $\mathrm{X}$ & $\mathrm{X}$ & $\mathrm{X}$ \\
\hline Events (adverse events) & & $\mathrm{X}$ & $\mathrm{X}$ & $\mathrm{X}$ & $\mathrm{X}$ & $\mathrm{X}$ \\
\hline
\end{tabular}

exclusion criteria are excluded (Table 3). Written informed consents are obtained from all the participants in a form approved by the IRB.

\section{Collection of Data}

The doctors in charge of each patient assess clinical data. Each doctor will input data into the Research Electronic Data Capture (REDcap), and then we will refer to those data, which is the browserbased electronic data capture (EDC) system. Table 1 shows the timing of data collection following patient enrollment. We will collect many phenotypes, including clinical, demographic, laboratory data, and events. Details are described in Supplemental Material. Events include new onset of general events, CVD, stroke, valvular/aortic, acute pancreatitis, and adverse events. In addition, we monitor clinical events specific to each intractable disease.

In this study, an independent staff member conducts data monitoring. The trial institution is monitored after patients' initial enrollment and every 6 months until each participant has a case report on file. The independent staff member monitors and reviews the trial database, and data queries and

\section{Advance Publication Journal of Atherosclerosis and Thrombosis}


Table 2. Inclusion criteria

1) Diagnosed either as HeFH, HoFH, type III hyperlipoproteinemia, familial chylomicronemia, sitosterolemia, CTX, LCAT deficiency, Tangier disease, APOA1 deficiency, and abetalipoproteinemia, according to the criteria set by Japan Atherosclerosis Society or the Committee on Primary Dyslipidemia under the Research Program on Rare and Intractable Disease of the Ministry of Health, Labor and Welfare of Japan.

2) Provide written informed consent.

Table 3. Exclusion criteria

1) Patients who refuse to consent to this study.

2) Considered inappropriate to participate by the doctors in charge.

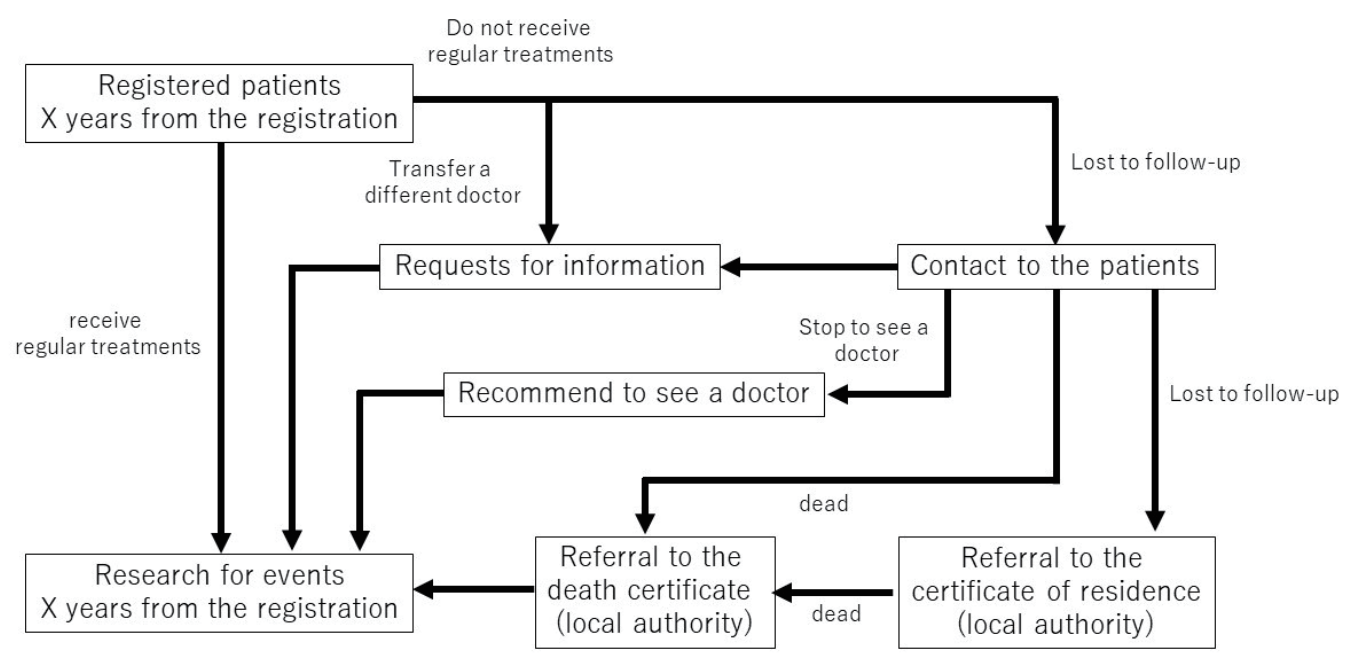

Fig. 2. Tracking of patients

cleaning will be raised if necessary.

\section{Outcomes}

The primary endpoint of this study is new CVD events, including myocardial infarction or angina pectoris, or acute pancreatitis. The secondary endpoint is death from any causes. Details are described in the Supplemental Material. The information of death and its cause are colleced to local authorities.

\section{Schedule of the Study}

Table 1 shows the schedule of this study. Follow-up visits are anticipated at each participated institute based on the conditions of the patients. Drop-out patients are to be traced by direct approach to the patients first and the maximum effort for further tracking (Fig.2). Nevertheless, we will collect those clinical variables at least once a year through the EDC system. Independent staff members monitor data at a certain interval (almost once a year). Whenever necessary, data queries will be sent to the investigators to check the data collection status and ensure the accuracy of the dataset.

\section{Statistical Analysis}

Mean values of continuous variables will be compared using Student's $t$-test for independent data variables, and median values will be compared using nonparametric Wilcoxon Mann-Whitney rank-sum test. Chi-square or Fisher's post-hoc tests will be used for categorical variables. A multivariable logistic regression model will be used to assess events and variables. Cox proportional hazard model will be used to assess relationships between all variables and events. Cumulative Kaplan-Meier survival curves starting at baseline will be constructed to compare times to the first events to find the meaningful variable. The statistical analysis was conducted using $\mathrm{R}$ statistics (https://www.r-project.org). $P$-values $<0.05$ were considered statistically significant.

\section{Data Availability Statement}

Dataset will not be available to public.

\section{Advance Publication Journal of Atherosclerosis and Thrombosis}




\section{Discussion}

The PROLIPID registry, organized by the Committee on Primary Dyslipidemia under the Research Program on Rare and Intractable Disease of the MHLW of Japan, is a nationwide, registry-based prospective, observational, multicenter cohort intended to understand clinical characteristics, genetic backgrounds, treatments, and prognosis of patients with primary dyslipidemias, including intractable diseases in Japan. This is a unique attempt, especially the registry, which will include rare lipid-related intractable diseases. This registry accepts all the patients with these intractable diseases treated in participating centers, trying to avoid selection bias, leading to illuminate a more detailed information of those disorders. We set the primary endpoint as new CVD events, and the secondary endpoint is all causes of death. Patients with $\mathrm{HeFH}, \mathrm{HoFH}$, type III hyperlipoproteinemia, sitosterolemia, LCAT deficiency, Tangier disease, and APOA1 deficiency are at increased risk for CVD because of their lifelong elevation of LDL cholesterol and/or extremely low HDL cholesterol. On the other hand, the patients with abetalipoproteinemia is quite cardioprotective while they suffer from various complications associated with deficiency of soluble vitamin. FH-associated mutation status is associated with an increased risk for $\mathrm{CVD}^{31)}$. Moreover, classical risk factors for CVD, including hypertension, smoking, or diabetes, have been associated with worsening the phenotype of $\mathrm{HeFH}$ although it is known as primary dyslipidemia ${ }^{32)}$. Given these facts, we try to assess those factors as well as genetic factors as well in order to understand the factors contributing to affect their phenotype fully. In addition, we set no exclusion criteria to include as many patients as possible, including those at younger ages, enabling us to know the age of onset of the clinical manifestations and complications in each disorder.

We emphasize that the inclusion of genetic information for all patients is one of our major strengths. Genetic testing can provide us an accurate diagnosis of these intractable diseases and risk stratifications for future CVD risk/other complications and help us support decision-making and facilitate cascade screening of those patients. Furthermore, this study provides us a useful insight into improving the current clinical diagnostic criteria of those diseases. Results of the genetic testing will be informed to the patients when necessary.

We hypothesize that patients with pathogenic mutations and/or other classical risk factors for CVD/ other complications are associated with an increased risk of CVD/other complications. We also expect that interventions manipulate their increased risk via genetic factors and/or classical risk factors according to treatment initiation and/or the intensiveness of the treatments. We hope to see more and more patients with these diseases are diagnosed accurately through this registry of these lipid-associated intractable diseases.

This study has several limitations. First, the final number of cases registered should vary by each disease, and that it is very difficult to assume the number of patients who will be enrolled. However, we set 1,000 as a target of the total number. Meanwhile, we will try to recruit consecutive patients with these disorders and enroll as many patients as possible. Second, blood testing will not be collected at follow-up, while medications will be followed for 5 years and 10 years. However, analyses will be performed based on baseline characteristics as well as medications as variables. Third, genotyping for all of the rare and intractable diseases included in this study is not covered by the public health insurance of Japan as of September 2021. In that sense, all the genotypes and its assessments will be left to each investigator's discretion. Fourth, there is no clear rationale for the duration of follow-up in this study because there is no data that we can estimate how many events will be occurred due to the rarity of these diseases. In that sense, we wanted to follow-up as much as possible, while the longest follow-up period the IRB can allow us to set is 10 years. Fifth, we do not use the universal definition of events, like an ICD 10 code. However, we will collect many information, such as date of onset, number of diseased arteries, change of electrocardiogram, elevation of cardiac enzymes, revascularization procedure, and coronary imaging in CVD. We believe that this information will make sure that the assessments of CVD are reliable.

\section{Acknowledgements} study.

We would like to thank the participants in this

\section{Competing Interests Statement}

Dr. Masatsune Ogura has received lecture fees from Amgen, Astellas Pharma Inc, and Kowa. Dr. Hidenori Arai has received lecture fees from MSD, Kowa, Daiichi Sankyo, Takeda, Pfizer, UCB, Sanofi, Astelas, and Otsuka. Dr. Mariko Harada-Shiba holds shares of Liid Pharma, has received lecture fees from Amgen, Astellas, and Sanofi, has received scholarship grants from Aegerion, Recordati, and Kaneka. Dr. 
Shun Ishibashi has received lecture fees from Kowa, and has received research grants from Ono Pharmaceutical Co.

\section{Funding Statement}

Ministry of Health, Labor and Welfare Research Grant for Research on Rare and Intractable Diseases of Japan supports this study.

\section{References}

1) Mabuchi H, Nohara A, Noguchi T, Kobayashi J, Kawashiri MA, Tada H, Nakanishi C, Mori M, Yamagishi M, Inazu A, Koizumi J; Hokuriku FH Study Group. Molecular genetic epidemiology of homozygous familial hypercholesterolemia in the Hokuriku district of Japan. Atherosclerosis, 2011; 214: 404-407

2) Sekijima Y, Koyama S, Yoshinaga T, Koinuma M, Inaba $Y$. Nationwide survey on cerebrotendinous xanthomatosis in Japan. J Hum Genet, 2018; 63: 271-280

3) Hu P, Dharmayat KI, Stevens CAT, Sharabiani MTA, Jones RS, Watts GF, Genest J, Ray KK, Vallejo-Vaz AJ. Prevalence of familial hypercholesterolemia among the general population and patients with atherosclerotic cardiovascular disease: a systematic review and metaanalysis. Circulation, 2020; 141: 1742-1759

4) Beheshti SO, Madsen CM, Varbo A, Nordestgaard BG. Worldwide Prevalence of Familial Hypercholesterolemia: Meta-Analyses of 11 Million Subjects. J Am Coll Cardiol, 2020; 75: 2553-2566

5) Mabuchi H. Half a century tales of familial hypercholesterolemia (FH) in Japan. J Atheroscler Thromb, 2017; 24: 189-207

6) Luirink IK, Wiegman A, Kusters DM, Hof $\mathrm{MH}$, Groothoff JW, de Groot E, Kastelein JJP, Hutten BA. 20-Year Follow-up of Statins in Children with Familial Hypercholesterolemia. N Engl J Med, 2019; 381: 15471556

7) Tada H, Okada H, Nomura A, Nohara A, Yamagishi M, Takamura M, Kawashiri MA. Prognostic impact of cascade screening for familial hypercholesterolemia on cardiovascular events. J Clin Lipidol, 2021; 15: 358-365

8) Yamamura T. Apolipoprotein E and Atherosclerosis. Doumyakukouka (Japanese), 1998; 25: 415-420

9) Hopkins PN, Brinton EA, Nanjee MN. Hyperlipoproteinemia type 3: the forgotten phenotype. Curr Atheroscler Rep, 2014; 16: 440

10) Harada-Shiba $M$, Arai $H$, Ishigaki $Y$, Ishibashi $S$, Okamura T, Ogura M, Dobashi K, Nohara A, Bujo H, Miyauchi K, Yamashita S, Yokote K and Working Group by Japan Atherosclerosis Society for Making Guidance of Familial H. Guidelines for diagnosis and treatment of familial hypercholesterolemia 2017. J Atheroscler Thromb, 2018; 25: 751-770

11) Nohara A, Tada H, Ogura M, Okazaki S, Ono K, Shimano H, Daida H, Dobashi K, Hayashi T, Hori M, Matsuki K, Minamino T, Yokoyama S, Harada-Shiba M; Committee on Primary Dyslipidemia under the Research
Program on Rare and Intractable Disease of the Ministry of Health, Labour and Welfare of Japan. Homozygous familial hypercholesterolemia. J Atheroscler Thromb, 2021; 28: 665-678

12) Luirink IK, Wiegman A, Kusters DM, Hof MH, Groothoff JW, de Groot E, Kastelein JJP, Hutten BA. 20-year follow-up of statins in children with familial hypercholesterolemia. N Engl J Med, 2019; 381: 15471556

13) Tada H, Okada H, Nomura A, Nohara A, Yamagishi M, Takamura M, Kawashiri MA. Prognostic impact of cascade screening for familial hypercholesterolemia on cardiovascular events. J Clin Lipidol, 2021 in press

14) Tada $H$, Nohara $A$, Inazu $A$, Sakuma $N$, Mabuchi $H$, Kawashiri MA. Sitosterolemia, hypercholesterolemia, and coronary artery disease. J Atheroscler Thromb, 2018; 25: 783-789

15) Tada H, Nomura A, Ogura M, Ikewaki K, Ishigaki Y, Inagaki K, Tsukamoto K, Dobashi K, Nakamura K, Hori M, Matsuki K, Yamashita S, Yokoyama S, Kawashiri MA, Harada-Shiba M; Committee on Primary Dyslipidemia under the Research Program on Rare and Intractable Desease of the Ministry of Health, Labour and Welfare of Japan. Diagnosis and management of sitosterolemia 2021. J Atheroscler Thromb, 2021; 28: 791-801

16) Koyama S, Sekijima Y, Ogura M, Hori M, Matsuki K, Miida T, Harada-Shiba M, Committee on Primary Dyslipidemia under the Research Program on Rare and Intractable Disease of the Ministry of Health, Labor and Welfare of Japan. Cerebrotendinous xanthomatosis: molecular pathogenesis, clinical spectrum, diagnosis, and disease-modifying treatments. J Atheroscler Thromb, 2021; 28: 905-925

17) Takahashi M, Okazaki H, Ohashi K, Ogura M, Ishibashi S, Okazaki S, Hirayama S, Hori M, Matsuki K, Yokoyama S, Harada-Shiba M, Committee on Primary Dyslipidemia under the Research Program on R, Intractable Disease of the Ministry of Health, Labor and Welfare of Japan. Current Diagnosis and Management of Abetalipoproteinemia. J Atheroscler Thromb, 2021; 28: 1009-1019

18) Okazaki H, Gotoda $T$, Ogura M, Ishibashi S, Inagaki K, Daida H, Hayashi T, Hori M, Masuda D, Matsuki K, Yokoyama S, Harada-Shiba M, Committee on Primary Dyslipidemia under the Research Program on Rare and Intractable Disease of the Ministry of Health, Labor and Welfare of Japan. Current diagnosis and management of primary chylomicronemia. J Atheroscler Thromb, 2021; 28: 883-904

19) Koseki M, Yamashita S, Ogura M, Ishigaki Y, Ono K, Tsukamoto K, Hori M, Matsuki K, Yokoyama S, HaradaShiba M, Committee on Primary Dyslipidemia under the Research Program on R, Intractable Disease of the Ministry of Health, Labor and Welfare of Japan. Current diagnosis and management of Tangier disease. J Atheroscler Thromb, 2021; 28: 802-810

20) Kuroda M, Bujo H, Yokote K, Murano T, Yamaguchi T, Ogura M, Ikewaki K, Koseki M, Takeuchi Y, Nakatsuka A, Hori M, Matsuki K, Miida T, Yokoyama S, Wada J, Harada-Shiba M; Committee on Primary Dyslipidemia under the Research Program on Rare and Intractable Disease of the Ministry of Health, Labour and Welfare of 
Japan. Current status of familial LCAT deficiency in Japan. J Atheroscler Thromb, 2021; 28: 679-691

21) Moriyama K, Sasaki J, Takada Y, Matsunaga A, Fukui J, Albers JJ, Arakawa K. A cysteine-containing truncated apo A-I variant associated with HDL deficiency. Arterioscler Thromb Vasc Biol, 1996; 16: 1416-1423

22) Huang W, Sasaki J, Matsunaga A, Nanimatsu H, Moriyama K, Han H, Kugi M, Koga T, Yamaguchi K, Arakawa K. A novel homozygous missense mutation in the apo A-I gene with apo A-I deficiency. Arterioscler Thromb Vasc Biol, 1998; 18: 389-396

23) Bujo H, Takahashi K, Saito Y, Maruyama T, Yamashita S, Matsuzawa Y, Ishibashi S, Shionoiri F, Yamada N, Kita T; Research Committeon Primary Hyperlipidemia of the Ministry of Health, Labour, and Welfare of Japan. Clinical features of familial hypercholesterolemia in Japan in a database from 1996-1998 by the research committee of the ministry of health, labour and welfare of Japan. J Atheroscler Thromb, 2004; 11: 146-151

24) Maruyama T, Yamashita S, Matsuzawa $Y$, Bujo $H$, Takahashi K, Saito Y, Ishibashi S, Ohashi K, Shionoiri F, Gotoda T, Yamada N, Kita T; Research Committee on Primary Hyperlipidemia of the Ministry of Health and Welfare of Japan. Mutations in Japanese subjects with primary hyperlipidemia--results from the Research Committee of the Ministry of Health and Welfare of Japan since 1996--. J Atheroscler Thromb, 2004; 11: 131145

25) Pérez de Isla L, Alonso R, Mata N, Fernández-Pérez C, Muñiz O, Díaz-Díaz JL, Saltijeral A, Fuentes-Jiménez F, de Andrés R, Zambón D, Piedecausa M, Cepeda JM, Mauri M, Galiana J, Brea Á, Sanchez Muñoz-Torrero JF, Padró T, Argueso R, Miramontes-González JP, Badimón L, Santos RD, Watts GF, Mata P. Predicting cardiovascular events in familial hypercholesterolemia: The SAFEHEART Registry (Spanish Familial Hypercholesterolemia Cohort Study). Circulation, 2017; 135: 2133-2144

26) Humphries SE, Cooper JA, Seed M, Capps N, Durrington PN, Jones B, McDowell IFW, Soran H, Neil HAW; Simon Broome Familial Hyperlipidaemia Register
Group. Coronary heart disease mortality in treated familial hypercholesterolaemia: Update of the UK Simon Broome FH register. Atherosclerosis, 2018; 274: 41-46

27) Yamashita S, Masuda D, Harada-Shiba M, Arai H, Bujo H, Ishibashi S, Daida H, Koga N, Oikawa $S$ and Group FS. Effectiveness and safety of lipid-lowering drug treatments in Japanese patients with familial hypercholesterolemia: Familial Hypercholesterolemia Expert Forum (FAME) Study. J Atheroscler Thromb, 2021 in press. doi: https: //doi.org/10.5551/jat.62764

28) Naito R, Daida H, Masuda D, Harada-Shiba M, Arai H, Bujo H, Ishibashi S, Koga N, Oikawa S, Yamashita S. Relation of Serum Lipoprotein(a) Levels to Lipoprotein and Apolipoprotein Profiles and Atherosclerotic Diseases in Japanese Patients with Heterozygous Familial Hypercholesterolemia: Familial Hypercholesterolemia Expert Forum (FAME) Study. J Atheroscler Thromb, 2021 in press. doi: http: //doi.org/10.5551/jat.63019

29) Ogura M, Harada-Shiba M, Masuda D, Arai $H$, Bujo $H$, Ishibashi S, Daida H, Koga N, Oikawa S, Yamashita S; FAME Study Group. Factors Associated with Carotid Atherosclerosis and Achilles Tendon Thickness in Japanese Patients with Familial Hypercholesterolemia: A Subanalysis of the Familial Hypercholesterolemia Expert Forum (FAME) Study. J Atheroscler Thromb, 2021 in press. doi: https: //doi.org/10.5551/jat.62925

30) https: //www.nanbyou.or.jp/entry/5354 (Last accessed at July 21, 2021)

31) Tada H, Kawashiri MA, Nohara A, Inazu A, Mabuchi $H$, Yamagishi M. Impact of clinical signs and genetic diagnosis of familial hypercholesterolaemia on the prevalence of coronary artery disease in patients with severe hypercholesterolaemia. Eur Heart J, 2017; 38: 1573-1579

32) Tada H, Kawashiri MA, Nohara A, Sakata K, Inazu A, Mabuchi H, Yamagishi M, Hayashi K. Remnant-like particles and coronary artery disease in familial hypercholesterolemia. Clin Chim Acta, 2018; 482: 120123 


\section{Supplemental Material}

$\begin{array}{ll}\text { Unit } & \text { Items } \\ \text { Entry } & \text { Research ID } \\ & \text { Date of registration } \\ \text { Confirmation if the patient is diagnosed as either of } & \text { target diseases } \\ & \text { Homozygous FH } \\ & \text { Heterozygous FH } \\ & \text { Type III hyperlipoproteinemia } \\ & \text { Familial chylomicronemia } \\ & \text { Sitosterolemia } \\ & \text { Cerebrotendinous xanthomatosis (CTX) } \\ & \text { Lecithin cholesterol acyltransferase (LCAT) deficiency } \\ & \text { Tangier disease } \\ & \text { Apolipoprotein A1 (APOA1) deficiency } \\ & \text { Abetalipoproteinemia } \\ & \text { Date of diagnosis } \\ & \text { Use the National aid for intractable diseases } \\ & \text { Registered in FAME study } \\ & \text { Informed consent }\end{array}$

Date of consent

Gender

Menstruation

The age of menopause

The year of birth

The month of birth

Age (at registration)

Demographics

Date of measurements

Height

Body weight

Waist circumference

choice/units

Yes/No

Yes/No

Yes/No

Yes/No

Yes/No

Yes/No

Yes/No

$\mathrm{Yes} / \mathrm{No}$

Yes/No

Yes/No

Yes/No

1. Applications are accepted, 2. Applications are denied, 3. Applications are evaluated, 4. not apply Yes/No

1. Reject, 2. written informed consent (baseline analysis and follow-up), 3. written informed consent (only baseline analysis), 4. Provide the chance to opt out

Vision test

Vision

Systolic blood pressure (First time)

Diastolic blood pressure (First time)

Systolic blood pressure (Second time)

Diastolic blood pressure (Second time)

Physical findings

Physical findings (sitosterolemia)

Specific physical findings and symptoms (CTX)

Details of neurologic manifestation

Specific physical findings and symptoms (LCAT

deficiency)

Specific physical findings and symptoms (Tangier disease, 1. opacity corneae, 2. Enlarged tonsils (orange-color), 3. and APOA1 deficiency)

Specific symptoms (abetalipoproteinemia) digestive

$\mathrm{cm}$

$\mathrm{kg}$

$\mathrm{cm}$

Yes/No

$\mathrm{mmHg}$

$\mathrm{mmHg}$

$\mathrm{mmHg}$

$\mathrm{mmHg}$

1. Achilles tendon thickness, 2. xanthoma tuberosum, 3. xanthoma planum, 4. xanthoma striata palmaris, 5. eruptive xanthoma, 6. xanthelasma palpebrarum, 7 . other tendinous xanthomas, 8. arcus cornea, 9. other

1. splenomegaly

1. jaundice, 2. neurologic manifestation

1. cognitive impairment, 2. psychological symptom, 3 . cerebellar symptom, 4. pyramidal sign, 5. extrapyramidal sign, 6. seizures, 7. spinalsensory disturbance, 8. peripheral neuropathy, 9. other neuropathy

1. opacity corneae, 2 . edema hepatomegaly, 4. splenomegaly, 5. peripheral neuropathy Yes/No

\section{Advance Publication Journal of Atherosclerosis and Thrombosis}


Details of digestive symptom

Specific symptoms (abetalipoproteinemia) eye symptom

Details of eye symptom

Specific symptoms (abetalipoproteinemia) neuropathy Details of neuropathy

Untreated lipids

Blood tests
Total cholesterol

LDL cholesterol

HDL cholesterol

Triglycerides

Nutrition (infant, one year or under)

LDL cholesterol (the highest value in life)

LDL cholesterol (the lowest value in life)

HDL cholesterol (the highest value in life)

HDL cholesterol (the lowest value in life)

Triglycerides (the highest value in life)

Triglycerides (the lowest value in life)

Date of blood tests

Condition of blood tests

Total cholesterol (TC)

LDL cholesterol

HDL cholesterol

Triglycerides

Blood glucose

Insulin

75g OGTT

Insulin $(0 \mathrm{~min})$

Insulin (30 min)

Insulin (60 min)

Insulin (120 min)

Blood glucose (0 min)

Blood glucose (30 min)

Blood glucose (60 min)

Blood glucose (120 min)

HOMA-IR

Inslulinogenic index

BUN

$\mathrm{Cr}$

eGFR

WBC

$\mathrm{RBC}$

$\mathrm{Ht}$

Plt

Total bilirubin

Direct bilirubin

ALT

AST

$y$-GTP
1. vomiting, 2. abdominal distension, 3. impaired development, 4. chronic diarrhea, 5. steatorrhea, 6. others

Yes/No

1. night blindness, 2. color blindness, 3. low vision, 4 . blindness, 5 . visual field defect, 6. retinal pigment degeneration, 7 . other

Yes/No

1. reduced deep tendon reflex, 2. disturbance of vibration sense, 3. disturbance of position sense, 4 . Romberg's test positive, 5. dysmetria, 6. ataxia, 7. ataxic gait, 8. dysbasia, 9. spastic paralysis, 10. contracture of skeletal muscle, 11. dysphemia, 12. other spinocerebellar symptoms, 13 . autonomic symptom, 14. peripheral neuropathy, 15. other neuropathy, 16. other symptoms related to muscles $\mathrm{mg} / \mathrm{dl}$

$\mathrm{mg} / \mathrm{dl}$

$\mathrm{mg} / \mathrm{dl}$

$\mathrm{mg} / \mathrm{dl}$

1. mother's milk, 2. formula milk, 3. both, 4 . other $\mathrm{mg} / \mathrm{dl}$

$\mathrm{mg} / \mathrm{dl}$

$\mathrm{mg} / \mathrm{dl}$

$\mathrm{mg} / \mathrm{dl}$

$\mathrm{mg} / \mathrm{dl}$

$\mathrm{mg} / \mathrm{dl}$

1. Fasting ( $\geq 10$ hours), 2. other, 3. Unknown

$\mathrm{mg} / \mathrm{dl}$

$\mathrm{mg} / \mathrm{dl}$

$\mathrm{mg} / \mathrm{dl}$

$\mathrm{mg} / \mathrm{dl}$

$\mathrm{mg} / \mathrm{dl}$

$\mu \mathrm{IU} / \mathrm{mL}$

Yes/No

$\mu \mathrm{IU} / \mathrm{mL}$

$\mu \mathrm{IU} / \mathrm{mL}$

$\mu \mathrm{IU} / \mathrm{mL}$

$\mu \mathrm{IU} / \mathrm{mL}$

$\mathrm{mg} / \mathrm{dl}$

$\mathrm{mg} / \mathrm{dl}$

$\mathrm{mg} / \mathrm{dl}$

$\mathrm{mg} / \mathrm{dl}$

$\mathrm{mg} / \mathrm{dl}$

$\mathrm{mg} / \mathrm{dl}$

$\mathrm{mL} / \mathrm{min} / 1.73 \mathrm{~m}^{2}$

$/ \mu \mathrm{L}$

$/ \mu \mathrm{L}$

$\%$

$/ \mu \mathrm{L}$

$\mathrm{mg} / \mathrm{dl}$

$\mathrm{mg} / \mathrm{dl}$

IU/L

IU/L

IU/L

Advance Publication Journal of Atherosclerosis and Thrombosis 


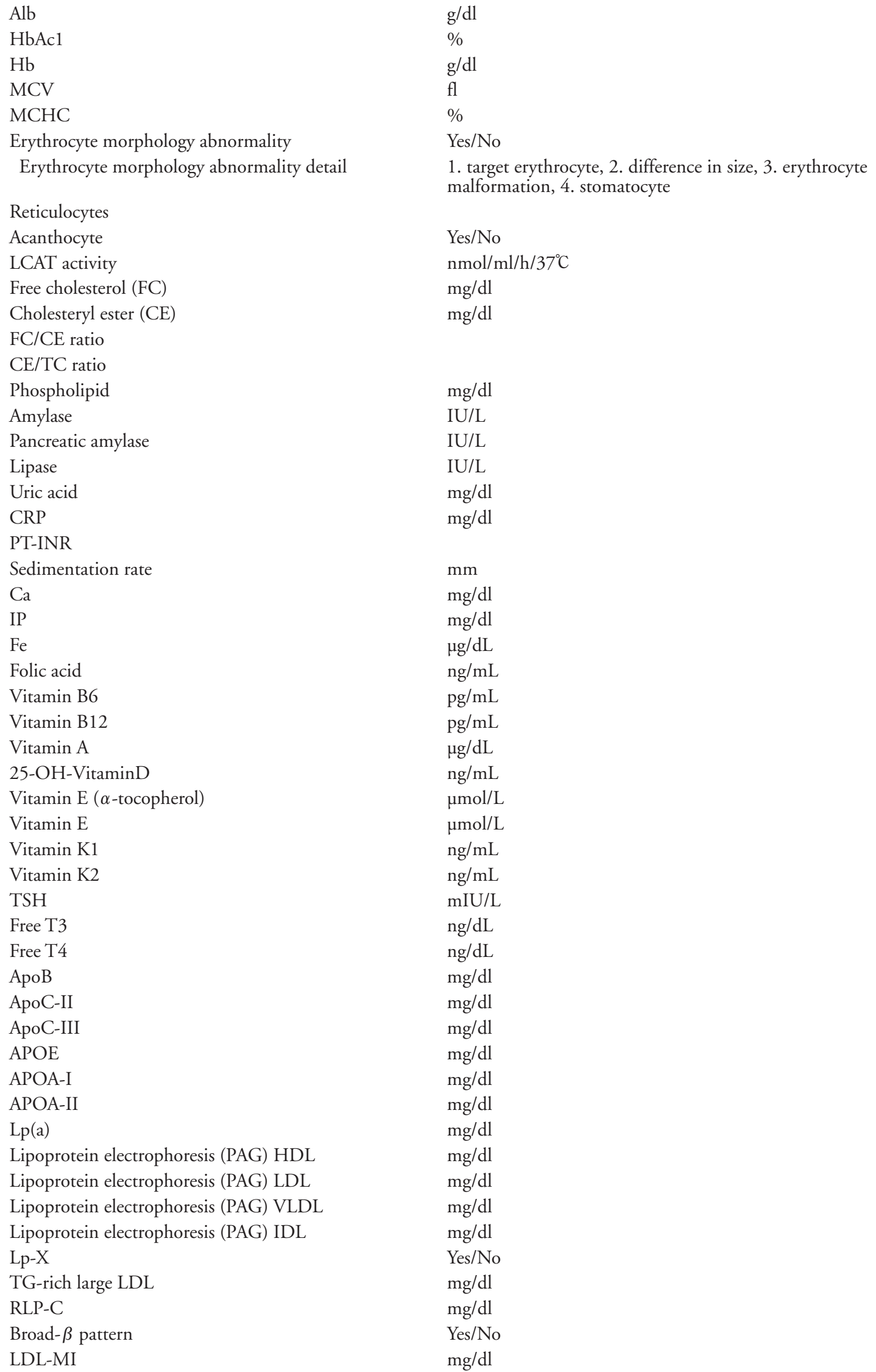

Advance Publication Journal of Atherosclerosis and Thrombosis 


\begin{tabular}{|c|c|c|}
\hline & LPL (pre-heparin) & $\mathrm{ng} / \mathrm{ml}$ \\
\hline & LPL (post-heparin) & $\mathrm{ng} / \mathrm{ml}$ \\
\hline & heparin unit & unit $/ \mathrm{kg}$ \\
\hline & Timing of blood test after heparin injection & $\min$ \\
\hline & LPL activity & $\mu \mathrm{mol} / \mathrm{mL} /$ hour \\
\hline & HL activity & $\mu \mathrm{mol} / \mathrm{mL} /$ hour \\
\hline & Timing of blood test after heparin injection & $\min$ \\
\hline & EPA & $\mu \mathrm{g} / \mathrm{mL}$ \\
\hline & $\mathrm{AA}$ & $\mu \mathrm{g} / \mathrm{mL}$ \\
\hline & EPA/AA ratio & \\
\hline & HPLC-HDL & $\mathrm{mg} / \mathrm{dl}$ \\
\hline & HPLC-LDL & $\mathrm{mg} / \mathrm{dl}$ \\
\hline & HPLC-IDL & $\mathrm{mg} / \mathrm{dl}$ \\
\hline & HPLC-VLDL & $\mathrm{mg} / \mathrm{dl}$ \\
\hline & HPLC-other & $\mathrm{mg} / \mathrm{dl}$ \\
\hline & HPLC-total cholesterol & $\mathrm{mg} / \mathrm{dl}$ \\
\hline & sitosterol & $\mu \mathrm{g} / \mathrm{ml}$ \\
\hline & cholestanol & $\mu \mathrm{g} / \mathrm{ml}$ \\
\hline & lathosterol & $\mu \mathrm{g} / \mathrm{ml}$ \\
\hline & campesterol & $\mu \mathrm{g} / \mathrm{ml}$ \\
\hline & X-ray of Achilles tendon & Yes/No \\
\hline Physical and imaging & Achilles tendon thickness (right) & $\mathrm{Mm}$ \\
\hline & Achilles tendon thickness (left) & $\mathrm{Mm}$ \\
\hline & $\mathrm{ABI}$ & Yes/No \\
\hline & ABI (right) & \\
\hline & ABI (left) & \\
\hline & ECG & Yes/No \\
\hline & Abnormality of ECG & Yes/No \\
\hline & Details of abnormality of ECG & \\
\hline & Carotid ultrasound & Yes/No \\
\hline & $\geq 70 \%$ stenosis (NASCET) & Yes/No \\
\hline & UCG & Yes/No \\
\hline & findings of UCG & 1. valvular disease, 2 . other \\
\hline & Abdominal ultrasound & Yes/No \\
\hline & Fatty liver & Yes/No \\
\hline & Cirrhosis & Yes/No \\
\hline & Other findings & Yes/No \\
\hline & Details of other findings of abdominal ultrasound & \\
\hline & Ophthalmoscopy & Yes/No \\
\hline & retinal pigment degeneration & Yes/No \\
\hline & Other findings & Yes/No \\
\hline & Details of other findings & \\
\hline & FMD & $\%$ \\
\hline & Visceral fat area & $\mathrm{cm}^{2}$ \\
\hline & Splenomegaly (CT or ultrasound) & Yes/No \\
\hline & Head MRI & Yes/No \\
\hline & atrophy & Yes/No \\
\hline & location of atrophy & \\
\hline & T2-FLAIR high intensity & Yes/No \\
\hline & location of T2-FLAIR high intensity & \\
\hline & Spinal MRI & Yes/No \\
\hline & atrophy & Yes/No \\
\hline & location of atrophy & \\
\hline & T2 high intensity & Yes/No \\
\hline
\end{tabular}


location of T2 high intensity

$\begin{array}{ll}\text { Electroencephalography } & \text { Yes/No } \\ \text { epilepsy } & \text { Yes/No } \\ \text { slow wave } & \text { Yes/No } \\ \text { Nerve conduction study } & \text { Yes/No } \\ \text { axon dysfunction } & \text { Yes/No } \\ \text { Demyelinisation } & \text { Yes/No } \\ \text { Bone density test } & \text { Yes/No } \\ \text { lumbar vertebra } & \\ \text { thigh bone } & \\ \text { Urine protein/Cr ratio } & \\ \text { Urine alb /Cr ratio } & \\ \text { Urine protein (24 hours) } & \\ \text { Gastrointestinal endoscopy } & \mathrm{g} \\ \text { snow white duodenum } & \text { Yes/No } \\ \text { Fes } / \text { No }\end{array}$

Family history of coronary artery disease $(<55 \mathrm{yr}$ male, 1. Yes, 2. No, 3. Unknown, 4. No investigation $<65$ yr female)

Family history

$$
\text { relationship }
$$

Family history of FH

relationship

Family history of hypertriglyceridemia

relationship

Consanguineous marriage

Family history of sitosterolemia

relationship

Family history of CTX

relationship

Family history of low HDL-C

relationship

Family history of low LDL-C

relationship

lipids in parent

lipids in child

lipids in sibling

lipids in grandparent

lipids in grandchild

Glucose intolerance

Medical history Diabetes

type

diabetic retinopathy (right)

other eye complications (right)

complications (right)

diabetic retinopathy (left)

other eye complications (left)
1. parent, 2. child, 3. sibling, 4. grandparent, 5. grandchild

1. Yes, 2. No, 3. Unknown, 4. No investigation 1. parent, 2. child, 3. sibling, 4. grandparent, 5. grandchild

1. Yes, 2. No, 3. Unknown, 4. No investigation 1. parent, 2. child, 3. sibling, 4. grandparent, 5. grandchild

1. Yes, 2. No, 3. Unknown, 4. No investigation

1. Yes, 2. No, 3. Unknown, 4. No investigation

1. parent, 2. child, 3. sibling, 4. grandparent, 5. grandchild

1. Yes, 2. No, 3. Unknown, 4. No investigation 1. parent, 2. child, 3. sibling, 4. grandparent, 5. grandchild

1. Yes, 2. No, 3. Unknown, 4. No investigation 1. parent, 2. child, 3. sibling, 4. grandparent, 5. grandchild

1. Yes, 2. No, 3. Unknown, 4. No investigation 1. parent, 2. child, 3. sibling, 4. grandparent, 5. grandchild

1. TC $<120 \mathrm{mg} / \mathrm{dl}$ or LDL-C $<70 \mathrm{mg} / \mathrm{dl}, 2$. TC $<50$ $\mathrm{mg} / \mathrm{dl}$ or LDL-C $<15 \mathrm{mg} / \mathrm{dl}$, 3. Unknown

1. TC $<120 \mathrm{mg} / \mathrm{dl}$ or LDL-C $<70 \mathrm{mg} / \mathrm{dl}, 2$. TC $<50$ $\mathrm{mg} / \mathrm{dl}$ or LDL-C $<15 \mathrm{mg} / \mathrm{dl}$, 3. Unknown

1. TC $<120 \mathrm{mg} / \mathrm{dl}$ or LDL-C $<70 \mathrm{mg} / \mathrm{dl}, 2$. TC $<50$ $\mathrm{mg} / \mathrm{dl}$ or LDL-C $<15 \mathrm{mg} / \mathrm{dl}$, 3. Unknown

1. TC $<120 \mathrm{mg} / \mathrm{dl}$ or LDL-C $<70 \mathrm{mg} / \mathrm{dl}, 2$. TC $<50$ $\mathrm{mg} / \mathrm{dl}$ or LDL-C $<15 \mathrm{mg} / \mathrm{dl}$, 3. Unknown

1. TC $<120 \mathrm{mg} / \mathrm{dl}$ or LDL-C $<70 \mathrm{mg} / \mathrm{dl}, 2$. TC $<50$ $\mathrm{mg} / \mathrm{dl}$ or LDL-C $<15 \mathrm{mg} / \mathrm{dl}$, 3. Unknown

Yes/No

Yes/No

1. type 1,2 . type 2,3 . other

A1 $\sim$ B5, or No

Yes/No

1. macular disease, 2. retinal detachment, 3. rubeotic glaucoma, 4. ischemic optic neuropathy, 5. photocoagulation, 6 . vitreous surgery

$\mathrm{A} 1 \sim \mathrm{B} 5$, or $\mathrm{No}$

Yes/No

\section{Advance Publication Journal of Atherosclerosis and Thrombosis}


complications (left)

CKD

PAD

Hypertension

TIA

Stroke

Ischemic stroke

Cerebral hemorrhage

Coronary artery disease

age of onset

intervention

AS

Supravalvular aortic stenosis

Dissecting aneurysm of aorta

Thoracic aortic aneurysm

max diameter of aorta (CT)

Abdominal aortic aneurysm

max diameter of aorta (CT)

Hypothyroidism

Other endocrine disease

Acute pancreatitis

Gallstone

Diagnostic ERCP

Therapeutic ERCP

Chronic pancreatitis

Malfusion of pancreaticobiliary ducts

Pancreas divisum

Pancreatic tumor

Hepatomegaly

Splenomegaly

Lipemia retinalis

Blood disorder

Details of blood disorder

Cataract

Chronic diarrhea

Osteoporosis

Prolonged Neonatal Jaundice

Neuropathy

Details of neuropathy

\section{Arthritis}

Hemorrhagic diathesis

Loss of vision

Blindness

Dialysis

Nephrotic syndrome

Cardiomyopathy

Fatty liver

Cirrhosis

Hepatocellular carcinoma

Other liver disease
1. macular disease, 2. retinal detachment, 3. rubeotic glaucoma, 4. ischemic optic neuropathy, 5.

photocoagulation, 6 . vitreous surgery

Yes/No

$\mathrm{Yes} / \mathrm{No}$

$\mathrm{Yes} / \mathrm{No}$

Yes/No

Yes/No

Yes/No

$\mathrm{Yes} / \mathrm{No}$

$\mathrm{Yes} / \mathrm{No}$

1. PCI, 2. CABG

$\mathrm{Yes} / \mathrm{No}$

Yes/No

$\mathrm{Yes} / \mathrm{No}$

Yes/No

$\mathrm{mm}$

Yes/No

$\mathrm{mm}$

Yes/No

Yes/No

Yes/No

Yes/No

Yes/No

Yes/No

Yes/No

Yes/No

Yes/No

Yes/No

Yes/No

Yes/No

$\mathrm{Yes} / \mathrm{No}$

Yes/No

Yes/No

$\mathrm{Yes} / \mathrm{No}$

Yes/No

Yes/No

Yes/No

1. cognitive impairment, 2. psychological symptom, 3 . cerebellar symptom, 4. pyramidal sign, 5 . extrapyramidal sign, 6. seizures, 7. Spinal sensory disturbance, 8. peripheral neuropathy, 9. other neuropathy

Yes/No

Yes/No

Yes/No

$\mathrm{Yes} / \mathrm{No}$

$\mathrm{Yes} / \mathrm{No}$

$\mathrm{Yes} / \mathrm{No}$

$\mathrm{Yes} / \mathrm{No}$

Yes/No

$\mathrm{Yes} / \mathrm{No}$

Yes/No

Yes/No

\section{Advance Publication Journal of Atherosclerosis and Thrombosis}


Medicine

Lifestyle habits

Details of other liver disease

Hyperthyroidism

Yes/No

Cancer

Yes/No

Details of cancer

Secondary causes of hypolipidemia

Yes/No

Details of secondary causes of hypolipidemia

Other specific disease

Yes/No

Details of other specific disease

Antihypertensive drug

Yes/No

class of antihypertensive drug

Antidiabetic drug (oral)

Yes/No

antidiabetic drug (injection)

class of antidiabetic drug (injection)

$\mathrm{Yes} / \mathrm{No}$

Antithrombotic drug

1. insulin, 2. GLP1

Immunosuppressive agent

Yes/No

$\mathrm{Yes} / \mathrm{No}$

class of immunosuppressive agent

details of immunosuppressive agent

Anticancer agent

1. azathioprine, 2. cyclosporine, 3 . other

class of anticancer agent

Antiinfective agent

class of antiinfective agent

Hormone preparations

class of hormone preparations

Treatments for neuropathy

class of treatments for neuropathy

Treatments fof acne vulgaris

Medications associated with pancreatitis

details of medications associated with pancreatitis

Medications associated with hypertriglyceridemia

details of medications associated with

hypertriglyceridemia

Statin

details of statin (class and dose)

Other lipid modifying medications

Yes/No

details of other lipid modifying medications (class and dose)

Smoking habits

1. current smoker, 2. former smoker, 3. never

peaces of smoking (daily)

duration of smoking

The age of quit smoking

Drinking

days of drinking (week)

1. daily, 2. quit, 3. never

The age of starting drinking

The age of quit drinking

duration of drinking

Types and amount of alcohol

FH pathogenic mutation

DNA LDLR pathogenic mutation

PCSK9 pathogenic mutation

LDLRAP1 pathogenic mutation

$A P O E$ genetic test

$A P O E$ genotype-1

APOE genotype-2

APOE phenotype test

APOE phenotype-1

1. Yes, 2. No, 3. No investigation

1. Yes, 2. No, 3. No investigation

1. Yes, 2. No, 3. No investigation

1. Yes, 2. No, 3. No investigation

Yes/No

Yes/No

Advance Publichentionpe-2 Journal of Atherosclerosis and Thrombosis 
Events (CVD)

LPL pathogenic mutation

$A P O C 2$ pathogenic mutation

GPIHBP1 pathogenic mutation

$L M F 1$ pathogenic mutation

$A P O A 5$ pathogenic mutation

$A B C G 5$ pathogenic mutation

$A B C G 8$ pathogenic mutation

CYP27A1 pathogenic mutation

$L C A T$ pathogenic mutation

$A B C A 1$ pathogenic mutation

$A P O A 1$ pathogenic mutation

ANGPTL3 pathogenic mutation

$S A R 1 B$ pathogenic mutation

Other pathogenic mutation

details of other pathogenic mutation

Date of investigation

Location of infarction

Type of angina pectoris

Date of onset

Number of diseased arteries

Change of ECG

details of ECG change

Elevation of CPK or TnT

PCI or CABG

coronary CT or MRI

Events (stroke)

Type of ischemic stroke

Date of onset

Symptom

Details of symptom

Imaging

type of imaging

results of imaging

Af

Thrombus in left atrium

Type

Events (valvular disease) Details of valvular disease

TAVI

Date of TAVI

Surgical treatment

Date of surgical treatment

Aortic disease

type of aortic disease

Intervention

Type of PAD

Imaging of PAD

Pancreatitis

Events (pancreatitis)

Date of onset

Symptom

Cause of pancreatitis

Type
1. Yes, 2. No, 3. No investigation

1. Yes, 2. No, 3. No investigation

1. Yes, 2. No, 3. No investigation

1. Yes, 2. No, 3. No investigation

1. Yes, 2. No, 3. No investigation

1. Yes, 2. No, 3. No investigation

1. Yes, 2. No, 3. No investigation

1. Yes, 2. No, 3. No investigation

1. Yes, 2. No, 3. No investigation

1. Yes, 2. No, 3. No investigation

1. Yes, 2. No, 3. No investigation

1. Yes, 2. No, 3. No investigation

1. Yes, 2. No, 3. No investigation

1. Yes, 2. No, 3. No investigation

1. anteroseptal, 2. lateral, inferior/posterior, 4. Unclassified

1. stable, 2. unstable, 3. coronary spasm angina, 4 . other

Yes/No

Yes/No

Yes/No

Yes/No

1. lacunar, 2. atherothrombotic, 3. cardioembolic, 4. unclassified

Yes/No

Yes/No

1. CT, 2. MRI, 3. other

Yes/No

Yes/No

1. aortic valve, 3. mitral valve, 3. tricuspid valve

Yes/No

Yes/No

Yes/No

Yes/No

1. CT, 2. MRI, 3. other

Yes/No

1. diabetes (new onset or worsening, 2. rhabdomyolysis, 3. dementia, 4. venous thrombosis, 5 . gallstone, 6 . other

Events (adverse)

Advance Publication Journal of Atherosclerosis and Thrombosis 